\title{
Reducing Broad-Spectrum Antibiotic Treatment of Simple Group A Streptococcal Infections to Reduce Harm to the Microbiome
}

\author{
Tyrone J. Sumibcay $^{1}$, Jannet J. Lee-Jayaram ${ }^{1,2}$, Loren G. Yamamoto ${ }^{1,2}$ \\ 1. Pediatrics, Kapi olani Medical Center for Women \& Children, Honolulu, USA 2. Pediatric Emergency Medicine, \\ University of Hawai'i, John A. Burns School of Medicine, Honolulu, USA
}

Corresponding author: Loren G. Yamamoto, loreny@hawaii.edu

\section{Abstract \\ Background}

Broad-spectrum antibiotics disrupt the human microbiome resulting in a greater risk of harmful, long-term conditions that impact human health. Group A streptococcal (GAS) infections can be treated with penicillin.

\section{Objective}

We examined the treatment of simple GAS infections to assess the use of broad-spectrum antibiotics.

\section{Methods}

Smart relational database extraction queries from January 1, 2016 to July 10, 2019 (3.6 years) of patients less than 22 years old in a 4-hospital system electronic medical record (EMR).

\section{Results}

We found 1778 non-ED outpatients and 873 ED patients with simple GAS infections who were not allergic to penicillin. A total of $75 \%$ and $44 \%$ of non-ED and ED patients were treated with broad-spectrum antibiotics, respectively $(p<0.001)$. Older patients were treated with penicillin alone more frequently than younger age groups $(\mathrm{p}<0.001)$.

\section{Conclusion}

These findings highlight opportunities for clinicians to reduce the utilization of broad-spectrum antibiotics for the treatment of simple GAS infections to reduce harm to the microbiome.

Review began 05/26/2021 Review ended 06/03/2021 Published 06/13/2021

\section{(๑) Copyright 2021}

Sumibcay et al. This is an open access article distributed under the terms of the Creative Commons Attribution License CC-BY 4.0., which permits unrestricted use, distribution, and reproduction in any medium, provided the original author and source are credited.
Categories: Pediatrics, Infectious Disease

Keywords: penicillin, streptococcal pharyngitis, microbiome, microbiota, broad-spectrum antibiotics

\section{Introduction}

The human microbiome is the microbial community that is crucial for human health and functioning. Disruption of the human microbiome, caused by a reduction in the diversity of its microorganisms that occurs with antibiotic treatment, can play a role in the development of harmful, long-term conditions that impact human health. Narrow-spectrum antibiotics disrupt the microbiome less than broad-spectrum antibiotics [1-5]. Antibiotic treatment of Group A streptococcal (GAS) infections, serves as an area where harm to the microbiome can be reduced since it can be treated with the narrow-spectrum antibiotic, penicillin, with $100 \%$ sensitivity. However, clinicians frequently prescribe broader spectrum antibiotics such as amoxicillin, cephalosporins, and clindamycin. While some might not consider amoxicillin to be a broadspectrum antibiotic, its coverage is clearly broader than that of penicillin and it is commonly cited as a common cause of pseudomembranous colitis along with clindamycin $[6,7,8,9]$. The purpose of this study is to characterize the antibiotics used to treat simple streptococcal throat infections and scarlet fever in children and to assess the degree of unnecessary broad-spectrum antibiotic use for these conditions.

Preliminary abstract of this work was presented at the 2019 Western Medical Research Conference in Carmel, CA on January 23, 2020. This abstract was published in the Journal of Investigative Medicine. An abstract of this work was submitted to the May 2020 Pediatric Academic Societies Meeting in Philadelphia, where it was accepted for presentation; however, the meeting was canceled due to COVID-19.

\section{Materials And Methods}

We performed a retrospective data extraction from the electronic medical record (EMR) system of a 4hospital health care system from January 1, 2016 to July 10, 2019 (3.6 years) of patients who were less than 
22 years old at the time of the encounter. This was accomplished by performing a stepwise smart relational database query from the EMR to extract all outpatient (office, clinic, acute care, urgent care, ED) encounters with International Classification of Diseases, 10th Revision, Clinical Modification (ICD-10-CM) diagnosis codes of acute streptococcal pharyngitis/tonsillitis or scarlet fever. This query included demographic information, disposition, medication allergies, medications (which included medications administered during the encounter, medications prescribed at the encounter, and medications prescribed within four days after the encounter), all encounter diagnoses, and all problem list diagnoses. Antibiotics were classified into groups according to the treatments administered during the encounter and prescribed within the next four days, which permitted us to capture changes in treatment in the four days immediately after the encounter. In some instances, a rapid streptococcal test could have been negative, but if this was followed by a positive culture result two days later, a prescription ordered within the four-day period was captured by this data query. The antibiotic classification was based on the broadest spectrum antibiotic administered or prescribed for the encounter with the assumption that the broadest spectrum drug harms the microbiome the most. The broadness sequence used was the following: amoxicillin < azithromycin < trimethoprim/sulfamethoxazole (TMP/SMX) < cephalosporins < amoxicillin/clavulanate < clindamycin. While this sequence is debatable, the number of prescriptions for azithromycin and TMP/SMX was low. If the patient was given intramuscular benzathine penicillin and prescribed amoxicillin, this encounter was classified as treatment with amoxicillin. If the patient was given intramuscular ceftriaxone but treated orally with penicillin, this encounter was classified as treatment with a cephalosporin.

Our target population was patients with simple streptococcal pharyngitis, tonsillitis, or scarlet fever (mild GAS infections). Thus, we excluded patients who were hospitalized during this encounter or left against medical advice. Patients were also excluded if they had a penicillin allergy or had co-infections or comorbidities that would require the use of broad-spectrum antibiotics, such as otitis media, sinusitis, impetigo, abscess, lymphadenitis, chronic/recurrent streptococcal infections, pneumonia, cellulitis, burns, urinary tract infection, renal disease (other than minimal change disease and microscopic hematuria), concurrent long-term corticosteroid treatment, indwelling catheters, cancers, congenital heart disease, valvular heart disease, chronic diseases associated with immunosuppression or bacterial infection recurrence.

\section{Results}

There were 3,632 encounters that met our initial search criteria in which the encounter diagnosis contained an ICD-10-CM code of streptococcal pharyngitis/tonsillitis or scarlet fever. We separated the groups into ED and non-ED (office, primary care, clinic, acute care, urgent care). The exclusions and age/gender distributions of the remaining encounters are described in Table 1. Penicillin allergy was more common in the non-ED group compared to the ED group (9.1\% versus $5.5 \%, \mathrm{p}=0.0002)$. Other exclusions were less common in the non-ED group compared to the ED group (18\% versus $22 \%, \mathrm{p}=0.001$ ).

\begin{tabular}{|c|c|c|c|c|}
\hline & All & 0 to 3 years & 4 to 12 years & 13 to 21 years \\
\hline Non-ED & 2424 & $263(11 \%)$ & 1467 (61\%) & $694(29 \%)$ \\
\hline Penicillin allergic & $220(9.1 \%)$ & 15 & 147 & 58 \\
\hline Other exclusions & 426 (18\%) & 91 & 253 & 82 \\
\hline Male inclusions & 918 & 80 & 559 & 279 \\
\hline Female inclusions & 860 & 77 & 508 & 275 \\
\hline ED & 1208 & $103(9 \%)$ & $761(63 \%)$ & $344(28 \%)$ \\
\hline Penicillin allergic & 67 (5.5\%) & 6 & 43 & 18 \\
\hline Other exclusions & $268(22 \%)$ & 29 & 162 & 77 \\
\hline Male inclusions & 438 & 37 & 305 & 96 \\
\hline Female inclusions & 435 & 31 & 251 & 153 \\
\hline
\end{tabular}

TABLE 1: Cohort demographics (3632 encounters).

Table 2 summarizes the antibiotic treatment groups for the cohort into penicillin (narrow spectrum), broadspectrum antibiotics, and no antibiotic treatment (despite a diagnosis of GAS pharyngitis/tonsillitis or scarlet fever in the encounter diagnosis). Non-ED patients were treated with broad-spectrum antibiotics more often than the ED patients $(75 \%$ versus $44 \%, \mathrm{p}<0.0001)$. In comparing the age groups of the combined cohort, the 0 to 3, 4 to 12 , and 13 to 21 -year-old age groups were treated with penicillin alone in $22 \%, 27 \%$, 


\section{Cureus}

and $37 \%$ of the encounters, respectively $(\mathrm{p}<0.0001)$.

\begin{tabular}{|c|c|c|c|c|c|}
\hline & Penicillin & $\{$ benzPCN\} & Broad spectrum & \{Broad spectrum + benzPCN\} & None \\
\hline Non-ED & $362(20 \%)$ & $\{227\}$ & 1337 (75\%) & $\{30\}$ & 79 (4\%) \\
\hline 0 to 3 years & 25 & $\{22\}$ & 124 & $\{5\}$ & 8 \\
\hline 4 to 12 years & 179 & $\{124\}$ & 843 & $\{14\}$ & 45 \\
\hline 13 to 21 years & 158 & $\{81\}$ & 370 & $\{11\}$ & 26 \\
\hline ED & $419(48 \%)$ & $\{329\}$ & $380(44 \%)$ & $\{50\}$ & 74 (8\%) \\
\hline 0 to 3 years & 24 & $\{19\}$ & 34 & $\{2\}$ & 10 \\
\hline 4 to 12 years & 254 & $\{205\}$ & 253 & $\{28\}$ & 49 \\
\hline 13 to 21 years & 141 & $\{105\}$ & 93 & $\{20\}$ & 15 \\
\hline Combined groups & $781(29 \%)$ & $\{556\}$ & 1717 (65\%) & $\{80\}$ & $153(6 \%)$ \\
\hline 0 to 3 years & 49 & $\{41\}$ & 158 & $\{7\}$ & 18 \\
\hline 4 to 12 years & 433 & $\{329\}$ & 1096 & $\{42\}$ & 94 \\
\hline 13 to 21 years & 299 & $\{186\}$ & 463 & $\{31\}$ & 41 \\
\hline
\end{tabular}

\section{TABLE 2: Antibiotic treatment groups by age group.}

Long-acting benzathine penicillin (benzPCN) was mostly given as monotherapy in the penicillin group, but sometimes it was given concurrently with penicillin and broad-spectrum oral antibiotics. These numbers are in subset brackets \{\} because they are a subset of and included in the column to its left.

Table 3 summarizes the broad-spectrum antibiotic groups prescribed. Within this group, amoxicillin comprised $88 \%$ of the non-ED patients, and $79 \%$ of the ED patients $(p=0.005)$. The other antibiotics were used less frequently, but it was noticeable that azithromycin was prescribed $7.3 \%$ in the non-ED patients, and $3.7 \%$ in the ED patients $(\mathrm{p}=0.02)$. 


\begin{tabular}{|c|c|c|c|c|c|c|}
\hline & Amoxicillin & Cephalosporins & Amoxicillin/Clavulanate & Azithromycin & Clindamycin & TMP/SMX \\
\hline Non-ED & 1172 & 37 & 28 & 97 & 0 & 3 \\
\hline 0 to 3 years & 114 & 3 & 1 & 6 & 0 & 0 \\
\hline 4 to 12 years & 754 & 20 & 12 & 55 & 0 & 2 \\
\hline 13 to 21 years & 304 & 14 & 15 & 36 & 0 & 1 \\
\hline ED & 301 & 37 & 9 & 14 & 19 & 0 \\
\hline 0 to 3 years & 26 & 6 & 0 & 1 & 1 & 0 \\
\hline 4 to 12 years & 214 & 18 & 5 & 8 & 8 & 0 \\
\hline 13 to 21 years & 61 & 13 & 4 & 5 & 10 & 0 \\
\hline Combined groups & 1473 & 74 & 37 & 111 & 19 & 3 \\
\hline 0 to 3 years & 140 & 9 & 1 & 7 & 1 & 0 \\
\hline 4 to 12 years & 968 & 38 & 17 & 63 & 8 & 2 \\
\hline 13 to 21 years & 365 & 27 & 19 & 41 & 10 & 1 \\
\hline
\end{tabular}

TABLE 3: Broad-spectrum antibiotic treatment groups by age group.

TMP/SMX: Trimethoprim/sulfamethoxazole.

\section{Discussion}

Our data indicate that amoxicillin and other broad-spectrum antibiotics are commonly used to treat simple GAS infections. Since penicillin has a narrow spectrum and GAS is $100 \%$ sensitive to penicillin, broadspectrum antibiotic treatment is not necessary to treat GAS and it results in greater harm to the microbiome.

Interestingly, $6 \%$ of the cohort was not treated with antibiotics. It is known that simple GAS pharyngitis infections will usually resolve without antibiotic treatment. Prompt antibiotic treatment, however, shortens the clinical course of symptoms and reduces the risk of suppurative complications, contagiousness, and acute rheumatic fever [10].

Study limitations include the retrospective nature of the study which makes the identification of simple GAS infections difficult to confirm; however, the methodology of reviewing all ICD-10-CM encounter diagnoses and the problem list should have identified complicated cases most of the time (Table 1). In 13 instances (eight non-ED and five ED), a case in which a patient who was initially treated with penicillin for a simple GAS infection was given a new prescription for a broad-spectrum antibiotic, which might have been for a clinically valid reason (e.g., otitis media); however, we did not review the clinical encounter data for the subsequent prescriptions which could have been done after all the data was collated and summarized but it was not part of the initially approved protocol to enter the patient's chart. Such encounters could have been simply removed since they are not simple GAS infections; however, such patients sustained the broadspectrum antibiotic's harm to their microbiome, and thus, we classified such patients as broad spectrum. Although it could be that the study group contains some patients who were treated with broad-spectrum antibiotics based on clinical necessity, it is more than likely that most of these patients could have been treated with penicillin.

Penicillin allergies are very likely to be reliable from a medical history standpoint using this methodology; however, it is well known that most patients who report a history of penicillin allergy are not truly allergic. Such patients subject themselves to less effective antibiotics (e.g., macrolides) and antibiotics resulting in greater harm to the microbiome (e.g., clindamycin, cephalosporins) [11].

Penicillin can be given orally four times per day (QID) or as a single intramuscular injection of long-acting benzathine penicillin. The 1.2 million units of long-acting benzathine penicillin can currently be purchased for $\$ 313$, while 40500 -mg penicillin VK tablets (a 10-day course) can be purchased for $\$ 6$, and the 200-ml bottle of $250 \mathrm{mg} / 5 \mathrm{ml}$ penicillin VK can be purchased for $\$ 12$. Thirty $500 \mathrm{mg}$ amoxicillin tablets can be purchased for $\$ 6$ and the $100-\mathrm{ml}$ bottle of $400 \mathrm{mg} / 5 \mathrm{ml}$ amoxicillin can be purchased for $\$ 5$ [12].

Long-acting benzathine penicillin injections were given frequently in both the non-ED and the ED cohorts. 
Of the 362 non-ED and the 419 ED patients treated with penicillin alone, 227 (63\%) and 329 (79\%) were given benzathine penicillin, respectively. Of the 1337 non-ED patients and $380 \mathrm{ED}$ patients treated with broad-spectrum antibiotics, 30 and 50 were also given benzathine penicillin, respectively. Benzathine penicillin must be refrigerated, and although it could be pre-purchased by office practices, it is generally obtained from a pharmacy.

Benzathine crystalline penicillin intramuscular injections are painful [10]. They are also viscous and opaque increasing the risk of intravascular injection [13]. Benzathine penicillin is known to be effective, but its duration of roughly 14 days or longer [14] exceeds the standard 10-day treatment duration. Prolonged or excessively long durations of antibiotics, even if it is a narrow-spectrum one, can prolong the adverse effect on the microbiome. In patients who are anticipated to complete a 10-day course of oral penicillin sufficiently, the additional pain, risk, and high cost of long-acting benzathine penicillin should be reconsidered.

Amoxicillin has the advantage of three times per day (TID) dosing, and some clinicians will dose this twice per day (BID). Cephalexin is usually dosed QID, in which case there would be no dosing advantage over oral penicillin. Azithromycin is dosed once daily. Amoxicillin/Clavulanate and clindamycin are dosed TID or BID, but their main indication for treating GAS throat infections is for suspected recurrent GAS infections or a GAS carrier state. If these conditions were described in the problem list or the encounter diagnosis, then these cases should have been excluded.

While GAS is $100 \%$ sensitive to penicillins and cephalosporins, there is growing resistance to macrolides (azithromycin) and clindamycin. Additionally, GAS sometimes possesses inducible macrolide-lincosamidestreptogramin (MLS) resistance [15].

QID dosing for penicillin is a clinical disadvantage that must be weighed against its advantage of reducing harm to the microbiome. Liquid penicillin has the reputation of poor taste compared to the other antibiotics that are used, but this problem with taste does not apply to pills, tablets, and capsules. This factor might be the reason for the higher penicillin frequency in the older patients who can swallow pills, compared to the younger patients who require liquid antibiotic formulations. The clinical practice of prescribing broadspectrum antibiotics for GAS infections evolved in an era during which the importance of the microbiome was not appreciated as much as it is today. Now with its importance identified, there should be a better understanding and appreciation of the harm resulting from unnecessary broad-spectrum antibiotics. While some consider amoxicillin to be a standard treatment for simple GAS infection, this should be reconsidered since its coverage is unnecessarily broad.

\section{Conclusions}

Our findings highlight opportunities for clinicians to reduce the utilization of broad-spectrum antibiotics for the treatment of simple GAS infection to reduce harm to the microbiome, which is essential in maintaining human health. GAS is still $100 \%$ sensitive to penicillin which is available orally, and for patients anticipated to have issues with compliance/adherence, single-dose long-acting penicillin injection is a treatment option. Since most broad-spectrum antibiotic-treated patients were treated with amoxicillin and cephalosporins, changing to narrow-spectrum penicillin is a relatively simple practice change that most patients can tolerate while reducing harm to the microbiome.

\section{Additional Information}

\section{Disclosures}

Human subjects: Consent was obtained or waived by all participants in this study. Hawaii Pacific Health Research Institute issued approval HPHRI Study Number: 2019-049. The Institutional Official of Hawai $i$ Pacific Health determined this study be exempt from Institutional Review Board review using the guidelines set by the Office for Human Research Protections (45 CFR 46, 101(d)(4)). Animal subjects: All authors have confirmed that this study did not involve animal subjects or tissue. Conflicts of interest: In compliance with the ICMJE uniform disclosure form, all authors declare the following: Payment/services info: The first author, Tyrone Sumibcay, was supported by the Hawai`i Pacific Health (HPH) Summer Student Research Program (SSRP). As an SSRP student scholar, Tyrone Sumibcay was provided with research training, health care professional training, and a summer stipend of approximately $\$ 1000$ during this eight-week program. The HPH SSRP is not considered to be a third party in this instance since this research was initiated as part of the HPH SSRP. HPH SSRP does not require research publication, but it does encourage it. Financial relationships: Loren Yamamoto declare(s) royalties from Challenger Corporation. Challenger Corporation: Pediatric Radiology Challenger. Royalties total approximately $\$ 30$ per year. . Loren Yamamoto declare(s) non-financial support from Kapi olani Medical Center For Women \& Children. Editorial board member: American Journal of Emergency Medicine, Pediatric Emergency Care. Chief of Staff and Board of Directors member, Kapi 'olani Medical Center For Women \& Children, 2014-2020. Loren Yamamoto declare(s) Receipt of medical supplies from BSN Medical (Orthoglass). Orthoglass supplies for educational training of medical students in splinting. Non-monetary, supplies only. Loren Yamamoto declare(s) royalties from McGraw Hill Medical Education Publishing. Editor, Strange and Schafermeyer’s Pediatric Emergency Medicine, 5th 
edition. Royalties approximately $\$ 1400$ per year. Loren Yamamoto declare(s) a grant from Hawaii Pediatric Association Research \& Education Foundation. Research grants to cover research supplies totaling approximately $\$ 1000$. The submitted manuscript did not benefit from any of these research supplies. . Other relationships: All authors have declared that there are no other relationships or activities that could appear to have influenced the submitted work.

\section{Acknowledgements}

The first author of this study was supported by the Hawaii Pacific Health Summer Student Research Program.

\section{References}

1. Goulet O, Hojsak I, Kolacek S, et al.: Paediatricians play a key role in preventing early harmful events that could permanently influence the development of the gut microbiota in childhood. Acta Paediatr. 2019, 108:1942-1954. 10.1111/apa.14900

2. Luca M, Di Mauro M, Di Mauro M, Luca A: Gut microbiota in Alzheimer's disease, depression, and type 2 diabetes mellitus: the role of oxidative stress. Oxid Med Cell Longev. 2019, 2019:4730539. $10.1155 / 2019 / 4730539$

3. Nichols RG, Peters JM, Patterson AD: Interplay between the host, the human microbiome, and drug metabolism. Hum Genomics. 2019, 13:27. 10.1186/s40246-019-0211-9

4. Vlasova AN, Takanashi S, Miyazaki A, Rajashekara G, Saif LJ: How the gut microbiome regulates host immune responses to viral vaccines. Curr Opin Virol. 2019, 37:16-25. 10.1016/j.coviro.2019.05.001

5. Berg G, Rybakova D, Fischer D, et al.: Microbiome definition re-visited: old concepts and new challenges. Microbiome. 2020, 8:103. 10.1186/s40168-020-00875-0

6. PubChem [Internet]. Bethesda (MD): National Library of Medicine (US), National Center for Biotechnology Information; 2004. PubChem Compound Summary for CID 6249, Ampicillin. (2004). Accessed: March 16, 2021: https://pubchem.ncbi.nlm.nih.gov/compound/Ampicillin.

7. Andréjak M, Schmit JL, Tondriaux A: The clinical significance of antibiotic-associated pseudomenbranous colitis in the 1990s. Drug Saf. 1991, 6:339-349. 10.2165/00002018-199106050-00004

8. Amin NM: Antibiotic-associated pseudomembranous colitis. Am Fam Physician. 1985, 31:115-120.

9. Brook I: Pseudomembranous colitis in children. J Gastroenterol Hepatol. 2005, 20:182-186. 10.1111/j.14401746.2004.03466.x

10. American Academy of Pediatrics Committee on Infectious Diseases: 2018-2021 Report of the Committee on Infectious Diseases. Kimberlin DW, Long SS, Brady MT, Jackson MA (ed): American Academy of Pediatrics, Itasca, IL; 2018. 31st edition:754.

11. Au LC, Siu AM, Yamamoto LG: Cost and risk analysis of lifelong penicillin allergy . Clin Pediatr (Phila). 2019, 58:1309-1314. 10.1177/0009922819853014

12. Henry Schein Medical. (2020). Accessed: August 10, 2020: http://HenrySchein.com.

13. Weir MR: Intravascular injuries from intramuscular penicillin . Clin Pediatr (Phila). 1988, 27:85-90. 10.1177/000992288802700205

14. Gartlan WA, Rahman S, Reti K: Benzathine Penicillin. August 11, 2020. StatPearls Publishing, Treasure Island, Florida; 2020 .

15. Sanson MA, Macias OR, Shah BJ, Hanson B, Vega LA, Alamarat Z, Flores AR: Unexpected relationships between frequency of antimicrobial resistance, disease phenotype and emm type in group A Streptococcus. Microb Genom. 2019, 5:10.1099/mgen.0.000316 